\title{
Dilemma Continues - Abdominal Tuberculosis or Abdomininal Lymphoma
}

\author{
Ajaz Nabi Koul, Aadil Rafeeq Rather, Basharat Ah. Kasana \\ Department of Internal Medicine \& Infectious Diseases, Sheri-I-Kashmir Institute of Medical Sciences (SKIMS) \\ Srinagar J\&K, India
}

\begin{abstract}
Tuberculosis is a disease that has long been there since ages and is a threat as it was. Abdominal tuberculosis is espacially common in endemic regions and with its varying and non-specific presentation may mimic a variety of disorders ranging from benign to malignant. It has a concealing effect as well and tends to mask diseases that are of entirely different character. All of this creates a diagnostic dilemma for the clinician. we present a similar case which was as non-specific as it could be. We presented here a patient who after undergoing more than 3 colonoscopies, being labelled lymphoma twice, registered for chemotherapy and most of the conventional tubercular markers being negative, still turned out to be a case of abdominal tuberculosis. J Microbiol Infect Dis 2017; 7(3):156-159
\end{abstract}

Keywords: Tuberculosis, Lymphoma, Colonoscopy, Histopathology

\section{INTRODUCTION}

Tuberculosis is a disease which has affected mankind for many centuries and persists to be a global threat in both developing and developed countries. Mycobacterium tuberculosis (TB) infection affects nearly 10 million people a year and causes 1.5 million deaths. TB is common in the immunosuppressed population with $12 \%$ of all new diagnoses occurring in human immune deficiency virus (HIV)-positive patients [1].

Abdominal TB is one of the most prevalent forms of extra-pulmonary disease being present in $11 \%$ of tuberculosis patients. It has an insidious course like any other chronic infectious disease without any specific laboratory, radiological or clinical findings which lead to great difficulties in its diagnosis. As a result, the diagnosis of abdominal TB is still a challenge to the physician. We present the case of a patient who after undergoing a battery of investigations, being labelled lymphoma twice, subjected to more than three colonoscopies, turned out to be abdominal tuberculosis.

\section{CASE}

A 52 year old post-menopausal female, with no underlying co-morbidities, presented with complaints of generalized weakness, evening rise of temperature and abdominal discomfort for last two months, associated with night-sweats, weight loss and reduced appetite. Examination revealed pallor with pulse rate of $82 / \mathrm{min}$, blood pressure of $124 / 80 \mathrm{mmHg}$ and temperature of 990F. There was no lymphadenopathy.

Her systemic examination was unremarkable except mild tenderness in left lower abdomen. Routine blood workup revealed anemia $(\mathrm{Hb} 10.5$ $\mathrm{g} / \mathrm{dl}$ ) which was hypochromic-microcytic (MCV$75 \mathrm{fL} / \mathrm{cell}, \mathrm{MCH}-23 \mathrm{pg} / \mathrm{cell})$ with normal Liver/Kidney function tests. Chest $\mathrm{x}$-ray was normal. USG abdomen was done which revealed retroperitoneal lymphadenopathy with mild ascites. CT abdomen showed enlarged retroperitoneal and bilateral common iliac nodes with omental thickening and stranding. There was mild to moderate ascites as well. Asciticfluid analysis revealed SAAG of 0.51 , neutrophillic exudative, ADA of $10 \mathrm{U} / \mathrm{l}$. It was negative for malignant cells. Microscopy as well as culture of ascitic fluid for Acid-Fast bacillus was negative but PCR-MTB was positive.

CA19-9 and CEA levels were normal, while CA125 was $153.5 \mathrm{U} / \mathrm{ml}$. Sputum as well as Urine for AFB were negative. Gut screening was done which revealed a normal upper GI tract while Colonoscopy suggested an Ulcero-infiltrative 
lesion at $55 \mathrm{~cm}$. from anal verge. Histopathology of this lesion was suggestive of large cell anaplastic lymphoma. Patient was registered in medical oncology unit but a review of the said biopsy by some other center revealed acute inflammation only with no evidence of lymphoma. Staining of this specimen for AFB did not reveal any organism. Following two contradictory reports, colonoscopy was repeated with the same findings of an ulcero-infiltrative lesion near splenic flexure and biopsy was taken from multiple sites viz. ascending colon, rectum and splenic-flexure lesion. Histopathology of both ascending colon and rectal biopsies showed only acute and chronic inflammatory infiltrate with no evidence of lymphoma and that of splenic flexure lesion revealed some pleomorphic cells with high nucleocytoplasmic ratio but was not confirmatory of malignancy and a repeat biopsy was advised.

Colonoscopy was repeated third time with the same findings but this time histopathology report of the biopsy came out to be that of NonHodgkin's lymphoma with infiltration by atypical lymphocytes having irregular nuclear membranes, hyperchromatic nuclei and inconspicuous nucleoli. With lymphoma twice a histopathological diagnosis, whole body CT was done.

CECT Findings: Concentric wall thickening involving descending colon with enlarged left para-aortic/ aortocaval/ peripancreatic and mesenteric nodes. Soft-tissue density nodules in bilateral upper lobes of lung with showing some feeding-vessel sign, suggestive of metastasis and micronodules with tree-in-bud appearance in upper lobes of lung, suggestive of infective pathology.

Patient was operated. Segmental left colectomy with end-to-end colocolic anastomosis was done. Operative findings were: About $3-4 \mathrm{~cm}$ ulcero-proliferative lesion in descending colon. Multiple small whitish nodules over peritoneum of pelvis, omentum and gut, likely metastasis with Splenomegaly and ascites.

Final histopathological report of the entire specimen depicted epithelioid cell granulomas with Langhans type of giant cells and caseous necrosis. Features were consistent with chronic granulomatous pathology- tuberculosis. Sections from meso-colon and single node dissected also showed features of chronic granulomatous pathology. Omental biopsy also revealed features of chronic granulomatous pathology.

Patient was started on Anti-tubercular chemotherapy and after one month of treatment she was symptomatically better with improved appetite. Her anemia was reversed and repeat abdominal scan showed a decrease in the lymph node size and ascites was resolved.

\section{DISCUSSION}

Abdominal tuberculosis is again on rise all over the world with the resurgence of multidrug resistant TB. However, this topic is still restricted within a few paragraphs in the textbooks and the current knowledge of abdominal tuberculosis has to be updated. Sensitivity of various methods have already been speculated in previous studies without any serious conclusion. The clinical and laboratory features of abdominal tuberculosis are nonspecific and lead to the suspicion of only a chronic infectious disease. The vague character of symptoms has been previously defined in many studies [2,3]. Symptoms such as weight loss, night-sweats, anorexia, anemia, hepatosplenomegaly and an elevated LDH can be present in a variety of GI disorders.

The diagnosis of abdominal tuberculosis classically requires microbiological and culture confirmation of the mycobacterium-tuberculosis, whereas, the diagnosis can be established histopathologically in many studies [2,4]. However, microbiological isolation of the agent is very rare for patients with abdominal tuberculosis. Tuberculosis can mimic a multitude of gastrointestinal disorders. Can present as large and palpable intra-abdominal masses, usually arising from lymphadenopathy, which may mimic lymphomas and other malignancies.

Lymphoma and tuberculosis are commonly being misdiagnosed due to the similarities in presentation of constitutional symptoms, subjecting patients to incorrect treatment and potential harm. The lymph nodes are the most common sites of extrapulmonary tuberculosis; abdominal TB comes in as sixth [5-7]. On the other hand, Gl tract is the predominant site of extra-nodal lymphoma, with the Non-Hodgkin type (NHL) being the most common variety. 


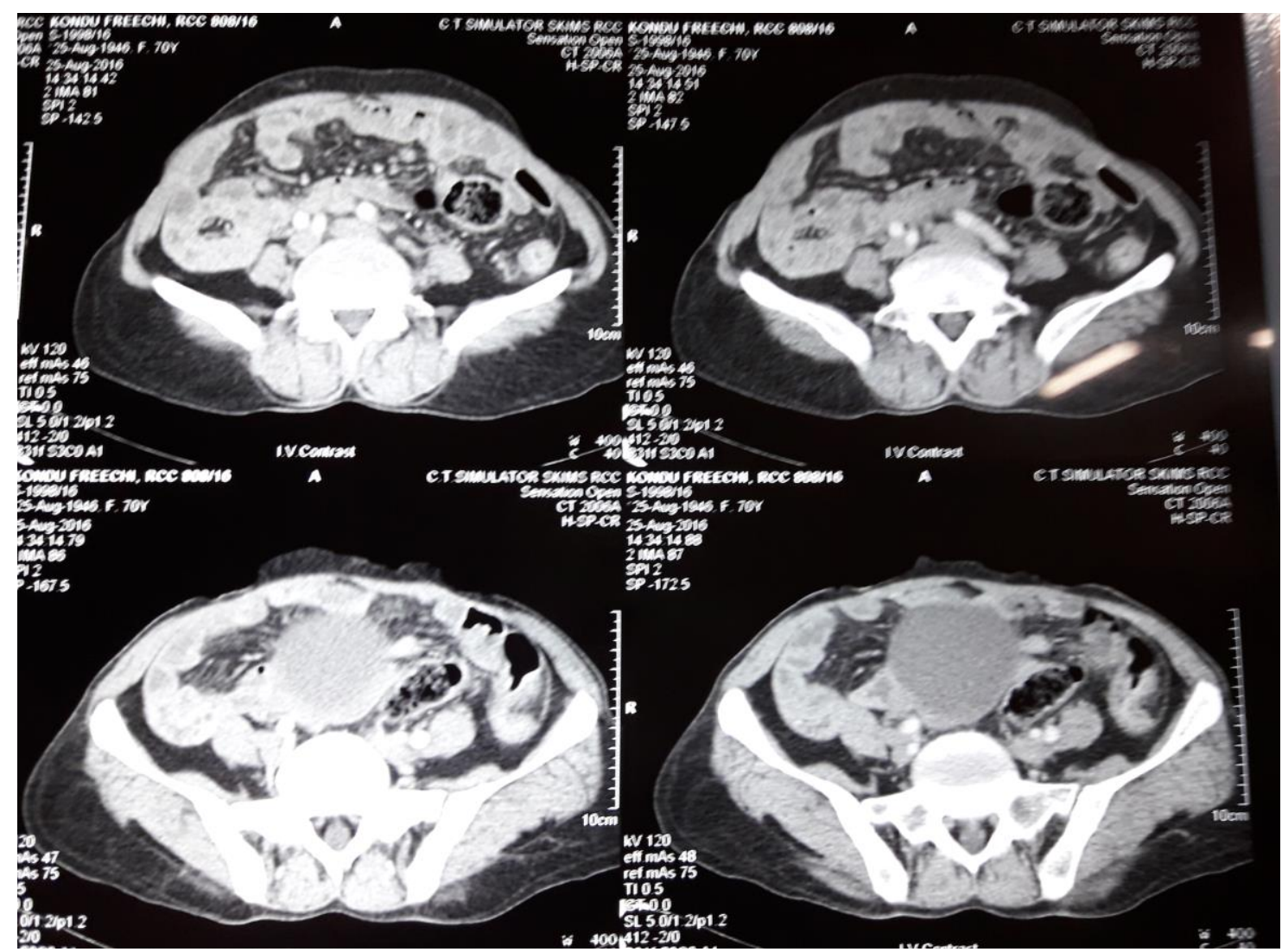

Figure 1. CECT Abdomen showing lymphadenopathy.

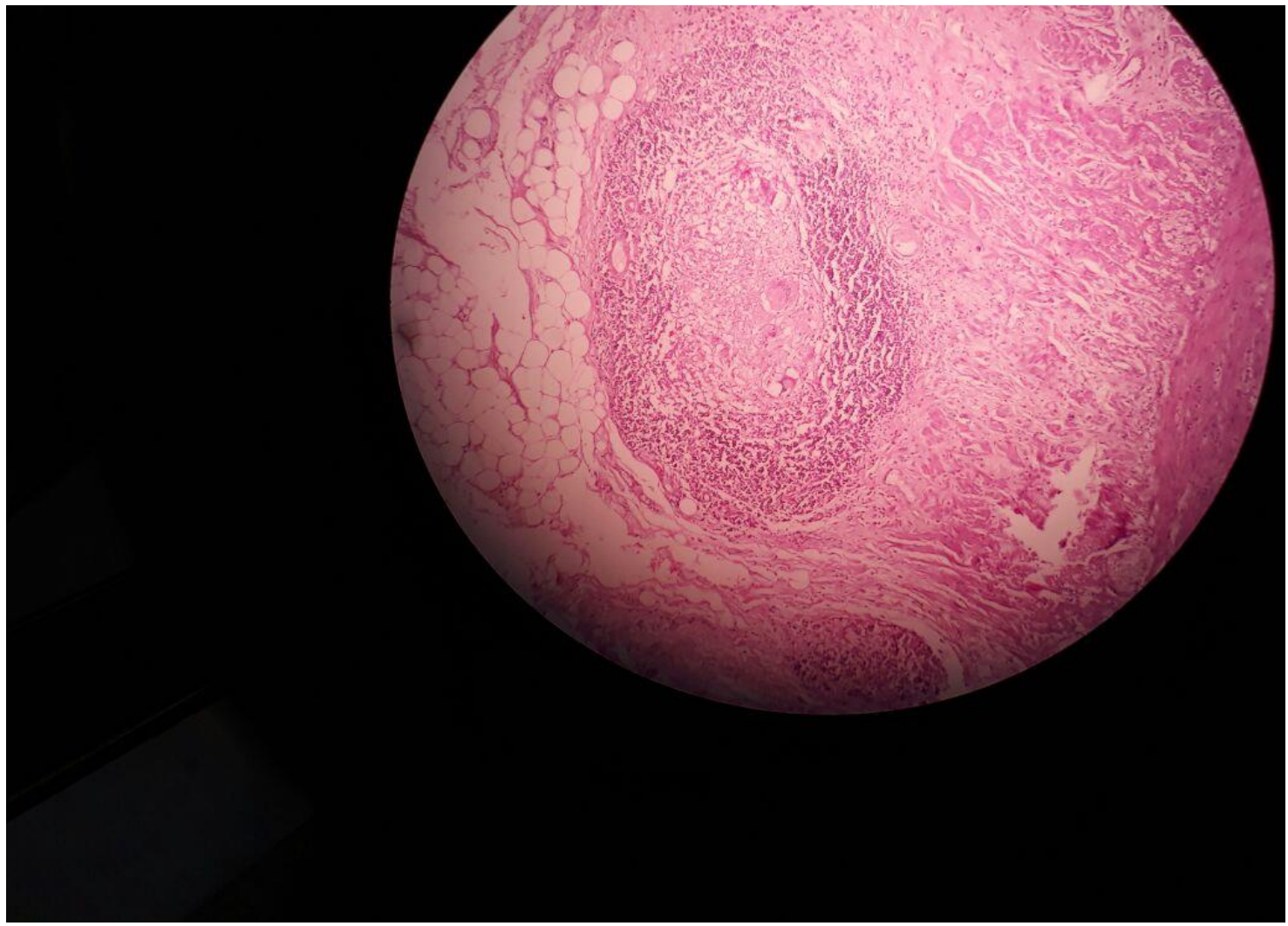

Figure 2. Colonic mucosa showing granulomas. 
TB and lymphoma may also share radiological features. In CT scan, there may be findings of polypoid masses or ulcerations, most frequently near the ileocecal valve; circumferential infiltration; a cavitary mass excavating into the mesentery; endoexoenteric tumors; mucosal nodularity; and fold thickening [8].

Several reports have described the coexistence of tuberculosis and non-Hodgkin lymphoma in lymph nodes [9]. Of importance, TB and lymphoma can be causatively related, through the well-established lymphoma related immunosuppression [10,11]. In the other direction, it has been reported that the risk of non-Hodgkin lymphoma is significantly increased (OR 1.8) in individuals with a history of TB [12]. The risk of non-Hodgkin lymphoma is increased in individuals with a history of severe forms of tuberculosis who have not received curative chemotherapy [13], and an underlying common susceptibility has been postulated.

Thus diagnosing abdominal tuberculosis or ruling it out of the probables in difficult cases is still a challenge to the physician. Like in our case, the clinical profile, laboratory findings and even the histopathology was in favour of malignancy. Patient was even registered for chemotherapy. But an aggressive investigative workup, which included a laparotomy, yielded a diagnosis which can be treated with simple and largely harmless drugs, for a very limited period of time.

In conclusion, tuberculosis is a great mimicker. It is also a disease that can be easily treated and controlled. Clinicians need to be aware of the myriad manifestations of TB and resist the temptation of premature diagnostic closure.

\section{ACKNOWLEDGMENTS}

\section{Declaration of conflicting interests}

The authors declare that they have no conflict of interest.

\section{Financial disclosure}

No financial support was received.

\section{REFERENCES}

1. Evans, RPT, Mourad MM, Dvorkin L, et al. Curr Infect Dis Rep 2016; 18: 45.

2. Al-Quorain AA, Satti MB, Al-Freihi HM, Al-Gindan YM, Al-Awad N. Abdominal tuberculosis in Saudi
Arabia: A clinicopathological study of 65 cases. Am J Gastroenterol 1993; 88: 75-79.

3. Lewis S, Field S. Intestinal and peritoneal tuberculosis. In: Rom WN, Garay S, eds. Tuberculosis. Little Brown and Company, Boston, USA; 1996; 585-597.

4. Bhargawa DK, Shrinivas S, Chopra P, Nijhawan S, Dasarathy S, Kushwaha AKS. Peritoneal tuberculosis: Laparoscopic patterns and its diagnostic accuracy. Am J Gastroenterol 1992; 87: 109-112.

5. Kushwaha R, Singhal S, Verma S. Case Report Disseminated tuberculosis mimicking lymphoma. Calicut Med J 2008; 6: e8.

6. Ayaslioglu E, Basar $H$, Duruyurek $N$, et al. Disseminated tuberculosis with lymphatic, splenic and scrotal abscesses: a case report. Cases J 2009; 2: 6995.

7. Tauro LF, Furtado HW, Aithala PS, et al. Primary Lymphoma of the Colon. Saudi J Gastroenterol 2009; 15: 279-282.

8. Dres $M$, Demoule A, Schmidt M, Similowski T Tuberculosis hiding a non-Hodgkin lymphoma "there may be more to this than meets the eye". Respir Med Case Rep 2012; 7: 15-16.

9. Bellido MC, Martino R, Martinez C, Sureda A, Brunet S. Extrapulmonary tuberculosis and nonHodgkin's lymphoma: coexistence in an abdominal lymph node. Haematologica 1995; 80(5):482e3.

10. Kaplan MH, Armstrong D, Rosen P. Tuberculosis complicating neoplastic disease. A review of 201 cases. Cancer 1974; 33(3):850e8.

11. Costa LJ, Gallafrio CT, Franca FO, del Giglio A. Simultaneous occurrence of Hodgkin disease and tuberculosis: report of three cases. South Med J 2004; 97(7):696e8.

12. Tavani A, La Vecchia C, Franceschi S, Serraino D, Carbone A. Medical history and risk of Hodgkin's and non-Hodgkin's lymphomas. Eur J Cancer Prev 2000; 9(1):59e64.

13. Askling J, Ekbom A. Risk of non-Hodgkin's lymphoma following tuberculosis. $\mathrm{Br} \mathrm{J}$ Cancer 2001; 84(1):113e5. 